\title{
Nutzung des Internets für die freiwillige Tätigkeit
}

\author{
Clemens Tesch-Römer \& Oliver Huxhold
}

\section{Kernaussagen}

Ein großer Teil der Engagierten nutzt das Internet im Rahmen ihrer freiwilligen Tätigkeit. Der Anteil der freiwillig engagierten Personen, die das Internet für ihre freiwillige Tätigkeit nutzen, beträgt 57,0 Prozent. Seit 2009 ist dieser Anteil nicht wesentlich gestiegen. Im Umkehrschluss bedeutet dies: Ein erheblicher Teil der Engagierten (43,o Prozent) nutzt das Internet für die freiwillige Tätigkeit nicht.

Die digitale Spaltung zeigt sich auch im Kontext des freiwilligen Engagements. Wie in anderen Bereichen des gesellschaftlichen Lebens gibt es auch im freiwilligen Engagement erhebliche Unterschiede nach Geschlecht, Alter und Bildung in der Nutzung des Internets. Freiwillig engagierte Männer nutzen das Internet anteilig häufiger für ihre freiwillige Tätigkeit als freiwillig engagierte Frauen, ältere Engagierte ab 65 Jahren nutzen es anteilig seltener als jüngere Altersgruppen und Engagierte mit hoher Bildung nutzen es anteilig häufiger als Engagierte mit niedriger Bildung.

Gerade bei den ältesten Engagierten hat der Anteil der Menschen, die das Internet für ihre freiwillige Tätigkeit nutzen, deutlich von 2004 bis 2019 zugenommen. Im Jahr 2004 nutzten nur 12,8 Prozent der Engagierten ab 65 Jahren das Internet, im Jahr 2019 sind es 48,o Prozent.

Nur ein geringer Teil der freiwillig Engagierten gibt an, dass ihre Tätigkeit überwiegend oder ausschließlich im Internet stattfindet. Der Anteil dieser ,Internet-Engagierten' ist klein und seit 2014 nicht gestiegen. Nur 2,6 Prozent aller freiwillig engagierten Personen üben ihre Tätigkeit überwiegend oder ausschließlich im Internet aus. Bei allen anderen freiwillig Engagierten, die das Internet nutzen, findet die freiwillige Tätigkeit nur teilweise im Internet statt. 
Aktiv-gestaltende Formen der Internetnutzung werden von einem großen Teil der Engagierten verwendet, die das Internet in ihrer freiwilligen Tätigkeit anwenden. Im Freiwilligensurvey 2019 wird nach fünf Formen der aktiv-gestaltenden Internetnutzung gefragt: Beteiligung an sozialen Netzwerken, Erstellung von Newslettern, Website-Betreuung, Werbung von Spenden sowie Angebote der Beratung und Lehre. Etwas mehr als die Hälfte der freiwillig Engagierten, die für ihre Tätigkeit das Internet verwenden, nennen mindestens eine dieser fünf aktiv-gestaltenden Formen der Internetnutzung. Am häufigsten genannt wird dabei die Nutzungsform der Beteiligung an sozialen Netzwerken. 


\subsection{Einleitung}

Die Digitalisierung ist ein zentraler gesellschaftlicher Trend des 21. Jahrhunderts. Digitale Informations- und Kommunikationstechnologien gewinnen in nahezu allen öffentlichen und privaten Lebensbereichen an Bedeutung. Laut Statistischem Bundesamt verfügen 90 Prozent der Privathaushalte in Deutschland über einen Internetzugang. Wiederum 90 Prozent der Personen ab 10 Jahren in diesen Haushalten nutzen das Internet täglich oder fast täglich. In den letzten zehn Jahren haben sowohl der Anteil der Haushalte mit einem Internetzugang als auch der Anteil der Personen, die das Internet nutzen, stetig zugenommen (Huxhold \& Otte 2019; Statistisches Bundesamt 2019).

Ein erhebliches Problem stellt die digitale Spaltung der Gesellschaft dar: Nicht alle gesellschaftlichen Gruppen haben in gleicher Weise Zugang zu digitalen Informations- und Kommunikationstechnologien. So gibt es deutliche Unterschiede im Zugang und in der Nutzung digitaler Technologien zwischen Menschen unterschiedlichen Alters, zwischen Frauen und Männern sowie zwischen Menschen mit unterschiedlichem Bildungshintergrund: Es sind etwas mehr Männer als Frauen und eher Personen mit hoher Bildung als Menschen mit niedriger Bildung online (Huxhold \& Otte 2019; Initiative D21 e. V. 2020; Statistisches Bundesamt 2019). Auch wenn für die Gruppe älterer Menschen eine gestiegene Internetnutzung verzeichnet werden kann, verfügen Personen ab 60 Jahren weiterhin seltener über einen Internetzugang und nutzen das Internet seltener als Jüngere (Huxhold \& Otte 2019; Initiative D21 e. V. 2020).

Die Digitalisierung zu gestalten und die digitale Spaltung der Gesellschaft zu überwinden, ist eine zentrale Herausforderung für Staat, Gesellschaft und Individuen. Dabei geht es darum, die Teilhabe aller Bevölkerungsgruppen in unterschiedlichen Bereichen des gesellschaftlichen Lebens zu gewährleisten. Auch im Bereich des freiwilligen Engagements werden die Möglichkeiten der Digitalisierung genutzt. Vielfach geht es bei der Digitalisierung des Engagements um die Unterstützung von freiwilligen Tätigkeiten, die weiterhin , analog' stattfinden, zum Beispiel die Terminkommunikation einer Übungsleiterin im Sportverein per E-Mail. In diesem Kontext wird häufig von der „Hybridisierung des Engagements“ gesprochen, also der Verknüpfung analoger und digitaler Tätigkeitskomponenten (Heinze et al. 2019: 75). Digitale Informations- und Kommunikationstechnologien sind hier Hilfsmittel, die dazu dienen, Aufgaben effizienter auszuführen (Hinz et al. 2014). Ebenso können unter die Digitalisierung des Engagements auch neue Formen des freiwilligen Engagements gefasst werden. Diese neuen Formen werden mit Schlagworten wie ,Engagement 4.0', digitales Engagement' oder ,Online-Volunteering' hervorgehoben (Deutscher Bundestag 2020; Heinze et al. 2019; Hinz et al. 2014). Dabei findet die freiwillige Tätigkeit ausschließlich oder überwiegend im Internet statt.

$\mathrm{Ob}$ und wie Engagierte das Internet für ihre freiwillige Tätigkeit nutzen, hängt davon ab, ob sie Zugang zum Internet haben und ihnen entsprechende Technik zur 
Verfügung steht. Vereine und andere Organisationen des freiwilligen Engagements sind mehr oder weniger gut mit digitaler Infrastruktur ausgestattet und bieten daher mehr oder weniger Möglichkeiten dafür, das Internet und andere digitale Kommunikationsdienste für die Tätigkeiten der freiwillig Engagierten zu nutzen. Je nach dem Ausmaß, zu dem das Internet und digitale Informations- und Kommunikationstechnologien in Organisationen genutzt werden, ist es auch für die Tätigkeiten freiwillig engagierter Personen von Bedeutung.

Die konkreten Aufgaben der freiwilligen Tätigkeit können unterschiedliche ,Di-

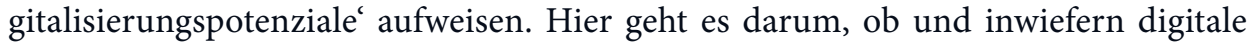
Elemente überhaupt für eine freiwillige Tätigkeit sinnvoll nutzbar sind. So finden Aufgaben der Öffentlichkeitsarbeit, wie die Beschaffung und Verbreitung von Informationen, bereits häufig digital statt. Bei Veranstaltungen und der Kommunikation mit Zielgruppen spielt hingegen auch eine analoge Zusammenarbeit der beteiligten Akteure eine wichtige Rolle (Heinze et al. 2019).

Schließlich richtet sich die Internetnutzung im Rahmen freiwilligen Engagements auch nach den individuellen Ressourcen der Engagierten. Neben der digitalen Spaltung im Internetzugang und in der Internetnutzung zeigen sich auch Unterschiede in der Bereitschaft sowie den Kompetenzen zur Internetnutzung. Die eigenen Kompetenzen im Umgang mit dem Internet und mit digitalen Informations- und Kommunikationstechnologien werden von Jüngeren, Personen mit höherer Bildung und Männern tendenziell höher eingeschätzt als von Älteren, Personen mit niedriger Bildung und Frauen. Ähnliche Muster zeigen sich auch für die Bereitschaft zur Nutzung digitaler Technologien (Initiative D21 e.V. 2020). Diese Unterschiede können eine weitere Erklärung für die Unterschiede in der Nutzung des Internets im Engagement zwischen Bevölkerungsgruppen sein.

Im Deutschen Freiwilligensurvey 2019 werden Engagierte zu drei Aspekten der Internetnutzung im Rahmen ihrer freiwilligen Tätigkeit befragt. Zunächst geht es allgemein darum, ob freiwillig Engagierte das Internet überhaupt für ihre Tätigkeit nutzen. In einem zweiten Schritt wird die Intensität der Internetnutzung erfasst. Das Internet kann für freiwilliges Engagement eine unterstützende Rolle darstellen. Mittlerweile lassen sich aber auch "neue Formen des Engagements im digitalen Zeitalter" erkennen (Deutscher Bundestag 2020: 34). Dieses ,Internet-Engagement' besteht in einer aktiven Beteiligung am Internet wie zum Beispiel der Bereitstellung von Inhalten in Blogs oder anderen sozialen Medien (Hoffmann et al. 2015).

Um tiefergehende Informationen über die freiwilligen Tätigkeiten, die online stattfinden, zu erhalten, werden im Freiwilligensurvey 2019 bedeutsame Formen der Internetnutzung abgefragt, bei denen es um die aktive Gestaltung im Internet geht. Aktive Beteiligung setzt gestaltende Mitarbeit voraus, also „etwas Eigenes beitragen, etwas schaffen oder ergänzen oder verändern" (Hoffmann et al. 2015: 26). In der entsprechenden Abfrage des Freiwilligensurveys werden aktiv-gestaltende Formen der Internetnutzung aufgeführt: Beteiligung an sozialen Netzwerken, Erstellung von Newslettern, Website-Betreuung, Werbung von Spenden sowie Angebote der 
Beratung und Lehre. Diese Formen der Internetnutzung gehen über einfache Internet-Anwendungen, wie Schreiben von E-Mails oder Recherche nach Informationen, deutlich hinaus. Ziel dieser Abfrage ist es, ein möglichst breites Spektrum von Nutzungsformen abzudecken, die aktiv und gestaltend im Internet ausgeführt werden.

In diesem Kapitel sollen folgende Fragen betrachtet werden:

a) Wie hoch ist der Anteil der Engagierten, die das Internet für ihre freiwillige Tätigkeit nutzen? Wie hat sich die Internetnutzung für die freiwillige Tätigkeit zwischen den Jahren 2004 und 2019 gewandelt? Welche Unterschiede finden sich im Anteil der Internetnutzung nach Geschlecht, Altersgruppen und Bildung?

b) In welchem Umfang nutzen Engagierte das Internet für ihre freiwillige Tätigkeit? Welcher Trend ist diesbezüglich zwischen 2014 und 2019 zu erkennen? Wie unterscheidet sich der Nutzungsumfang nach Geschlecht, Altersgruppen und Bildung?

c) Welche Formen der Internetnutzung werden im Rahmen der freiwilligen Tätigkeit genutzt? Wie unterscheiden sich die Formen der Internetnutzung nach Geschlecht, Altersgruppen und Bildung der Engagierten? Wie unterscheiden sich die Nutzungsformen nach dem Umfang der Internetnutzung für die freiwillige Tätigkeit?

\subsection{Daten und Indikatoren}

Die Ergebnisse des vorliegenden Kapitels beruhen auf den Daten des Deutschen Freiwilligensurveys (FWS) und werden gewichtet dargestellt. Die Gewichtung zielt darauf ab, die Repräsentativität zu verbessern, um verallgemeinerbare Aussagen treffen zu können. In den Veröffentlichungen zu den Erhebungen der Jahre 1999 bis 2014 wurden im Rahmen des Freiwilligensurveys bislang die Gewichtungsmerkmale Geschlecht, Alter, Bundesland sowie Gemeindegrößenklasse herangezogen. Diese Gewichtung wird nun um das Gewichtungsmerkmal der schulischen Bildung ergänzt. Personen aus unterschiedlichen Bildungsgruppen nehmen mit unterschiedlicher Wahrscheinlichkeit an Umfragen teil; daher sind verschiedene Bildungsgruppen in den Daten über- beziehungsweise unterrepräsentiert. Die Gewichtung inklusive schulischer Bildung wird in diesem Bericht auf die Daten aller fünf Erhebungswellen von 1999 bis 2019 angewendet. Die Engagementquoten fallen durch dieses Vorgehen für alle Erhebungswellen niedriger aus als bisher berichtet; es ändert sich somit das Niveau, der Trend bleibt jedoch im Wesentlichen bestehen. Die Gewichtung der Daten inklusive Bildung wirkt sich auch auf andere Ergebnisse aus.

Alle Ergebnisse werden auf statistische Signifikanz getestet (verwendet wird ein Signifikanzniveau von $\mathrm{p}<0,05$ ). Die Prüfung auf statistische Signifikanz zeigt an, ob ein anhand der Daten des Freiwilligensurveys gefundener Unterschied mit großer Wahrscheinlichkeit auch in der Grundgesamtheit, also beispielsweise in der Wohn- 
bevölkerung Deutschlands, vorhanden ist (statistisch signifikant) oder nur zufällig in den Daten vorliegt (statistisch nicht signifikant).

Die dargestellten Ergebnisse fußen auf der Befragung des Freiwilligensurveys 2019, die von März bis November 2019 erfolgte. Somit ist es mithilfe der Daten des Freiwilligensurveys 2019 nicht möglich, Aussagen über spätere Entwicklungen wie beispielsweise die Auswirkungen der Corona-Pandemie auf das freiwillige Engagement zu tätigen.

Internetnutzung. Um die Bedeutung des Internets für das freiwillige Engagement zu erfassen, werden alle engagierten Personen nach der Nutzung des Internets für ihre freiwillige Tätigkeit (einzige Tätigkeit oder zeitaufwendigste Tätigkeit bei mehreren Tätigkeiten) gefragt:

Nutzen Sie für Ihre Tätigkeit das Internet?

Antwortkategorien: ja; nein

Intensität der Internetnutzug. Freiwillig engagierte Personen, die angeben, in ihrer freiwilligen Tätigkeit das Internet zu nutzen, wird eine Folgefrage gestellt:

Findet Ihre Tätigkeit ausschließlich oder überwiegend oder nur teilweise im Internet statt? Antwortkategorien: ausschließlich; überwiegend; nur teilweise

Für die Darstellung der Ergebnisse werden die Kategorien ,ausschließlich und ,überwiegend' zusammengefasst, zudem wird auch der Anteil der Personen ausgewiesen, die das Internet nicht für ihre Tätigkeit nutzen.

Aktiv-gestaltenden Formen der Internetnutzung. An alle freiwillig engagierten Personen, die angeben, in ihrer freiwilligen Tätigkeit das Internet zu nutzen, wird eine Frage zur Form der Internetnutzung gestellt. Dabei werden fünf ausgewählte Formen der aktiv-gestaltenden Internetnutzung vorgelegt. In der Beantwortung der Frage sind Mehrfachnennungen möglich:

Ich nenne Ihnen nun verschiedene Formen der Internetnutzung. Bitte sagen Sie mir bei jeder Form, ob Sie diese für Ihre Tätigkeit nutzen.

(1) Beteiligen Sie sich an sozialen Netzwerken, Blogs, Foren oder Wikis?

(2) Betreuen Sie die Homepage eines Vereins oder einer Organisation?

(3) Erstellen Sie Newsletter oder Onlineberichte?

(4) Organisieren Sie Fund- oder Friendraising, werben Sie also Geld-, Sachspenden oder Engagierte?

(5) Bieten Sie Lehre, Beratung oder Expertisen im Internet an?

(6) Sonstiges?

Mehrfachnennung möglich; Antwortkategorien (jeweils): ja; nein 
Bei der Beantwortung der Frage nach Formen der Internetznutzung im Rahmen der freiwilligen Tätigkeit lassen sich zwei Gruppen von Personen unterscheiden. Personen in der ersten Gruppe geben an, mindestens eine der aktiv-gestaltenden Internetnutzungsformen (1) bis (5) in ihrer freiwilligen Tätigkeit zu verwenden - das sind 53,9 Prozent der Engagierten, die bei ihrer freiwilligen Tätigkeit das Internet nutzen. Personen in der zweiten Gruppe geben keine der Internetnutzungsformen (1) bis (5) an - das sind 46,1 Prozent der Engagierten, die in ihrer freiwilligen Tätigkeit das Internet nutzen. Die zweite Gruppe setzt sich zusammen aus Personen, die ausschließlich die Option (6) ,Sonstiges' angeben (20,3 Prozent der Engagierten, die das Internet in ihrer freiwilligen Tätigkeit nutzen) sowie aus Personen, die keine der Optionen (1) bis (6) angeben (25,9 Prozent der Engagierten, die das Internet in ihrer freiwilligen Tätigkeit nutzen). Demnach wird diese zweite Gruppe wahrscheinlich aus Personen gebildet, die das Internet aktiv-gestaltend nutzen (außerhalb der hier genannten fünf Formen) sowie aus Personen, die für ihre freiwillige Tätigkeit einfache Internet-Anwendungen verwenden (zum Beispiel Versand von E-Mails, Recherche nach Informationen).

\subsection{Nutzung des Internets für die freiwillige Tätigkeit}

In der ersten Welle des Freiwilligensurveys im Jahr 1999 wurde nicht nach der Nutzung des Internets für die freiwillige Tätigkeit gefragt. Erst Daten der zweiten Befragung lassen Aussagen über die Bedeutung des Internets für das freiwillige Engagement zu. Im Jahr 2004 nutzten 39,2 Prozent der freiwillig engagierten Personen das Internet für ihre Tätigkeit (Abbildung 11-1). Zwischen 2004 und 2009 gab es einen erheblichen und statistisch signifikanten Anstieg des Anteils der Engagierten, die das Internet für ihre Tätigkeit nutzen. Die entsprechende Quote stieg um etwa 16 Prozentpunkte auf 55,8 Prozent. Seitdem hat die Nutzung des Internets für die freiwillige Tätigkeit nicht merklich zugenommen. Von 2009 bis 2019 liegt der Anteil von Personen, die für ihre freiwillige Tätigkeit das Internet nutzen, bei 55,7 bis 57,0 Prozent.

Allerdings nutzt ein erheblicher Anteil der Engagierten das Internet nicht für die freiwillige Tätigkeit. Im Jahr 2004 waren dies 60,8 der freiwillig engagierten Personen. Dieser Anteil sank zwar auf 43,o Prozent im Jahr 2019 (Abbildung 11-1), ist aber angesichts der weitverbreiteten Überzeugung, dass die Digitalisierung alle Lebensbereiche durchziehe und auch für das freiwillige Engagement hohe Bedeutung habe, erstaunlich hoch.

Bei der Internetnutzung im freiwilligen Engagement gibt es deutliche Unterschiede zwischen den Geschlechtern, die sich aber seit 2004 verringert haben (Abbildung 11-2). Im Jahr 2004 nutzten 31,5 Prozent der Frauen und 45,9 Prozent der Männer das Internet für ihre freiwillige Tätigkeit. Im Jahr 2019 nutzen 53,8 Prozent der Frauen und 60,1 Prozent der Männer das Internet für ihre freiwillige Tätigkeit. Entsprechend dem allgemeinen Trend stieg die Internetnutzung von Frauen und Männer zwischen 
Abbildung 11-1 Anteile freiwillig engagierter Personen, die das Internet für ihre freiwillige Tätigkeit nutzen, im Zeitvergleich 2004-2019 (Basis: alle Engagierten)

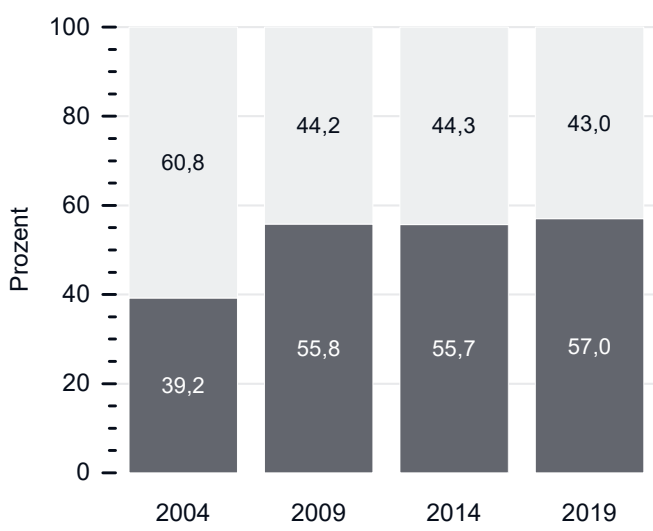

Keine Nutzung des Internets für die freiwillige Tätigkeit

Nutzung des Internets für die freiwillige Tätigkeit

Quelle: FWS, gewichtet, eigene Berechnungen (DZA). Basis: alle Engagierten. FWS 2004 ( $n=5.141)$, FWS 2009 ( $n=7.001)$, FWS $2014(n=12.421)$, FWS 2019 ( $n=11.925$ ). Im Jahr 1999 wurde Internetnutzung für die freiwillige Tätigkeit nicht erhoben.

2004 unterscheidet sich statistisch signifikant von 2009, 2014 und 2019. Die Jahre 2009, 2014 und 2019 unterscheiden sich nicht statistisch signifikant voneinander.

Abbildung 11-2 Anteile freiwillig engagierter Personen, die das Internet für ihre freiwillige Tätigkeit nutzen, nach Geschlecht, im Zeitvergleich 2004-2019 (Basis: alle Engagierten)

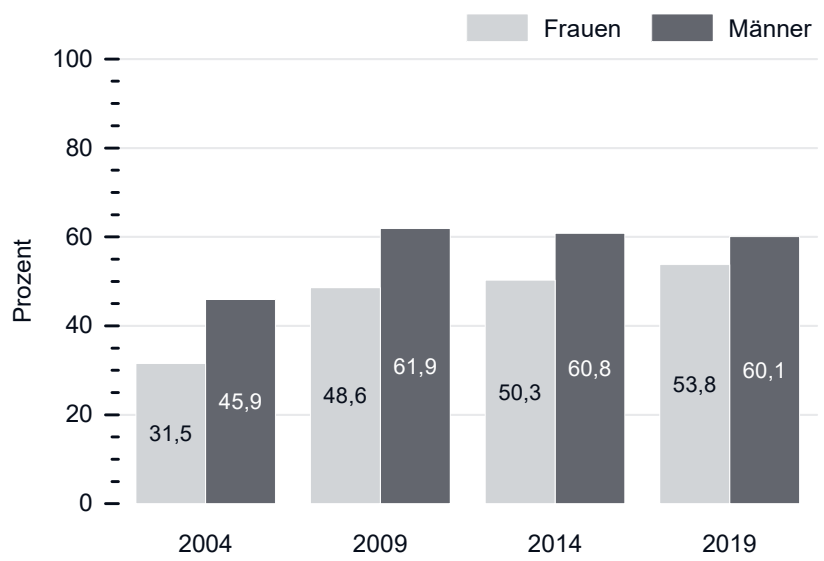

Quelle: FWS, gewichtet, eigene Berechnungen (DZA). Basis: alle Engagierten. FWS 2004 ( $n=5.141)$, FWS 2009 ( $n=7.001$ ), FWS 2014 ( $n=12.421$ ), FWS 2019 ( $n=11.925)$. Im Jahr 1999 wurde Internetnutzung für das freiwillige Engagement nicht erhoben.

Die Geschlechterunterschiede sind in jedem Jahr statistisch signifikant.

Frauen im Zeitvergleich: Statistisch signifikante Unterschiede zwischen 2004 zu allen anderen Jahren. Statistisch signifikante Unterschiede zwischen 2009 und 2019 sowie zwischen 2014 und 2019. Kein statistisch signifikanter Unterschied zwischen 2009 und 2014.

Männer im Zeitvergleich: Statistisch signifikante Unterschiede nur zwischen 2004 zu allen anderen Jahren. 
2004 und 2009 deutlich und statistisch signifikant an. Seit 2009 hat sich das Niveau bei Männern nicht verändert. Bei Frauen gibt es einen moderaten Zuwachs im gleichen Zeitraum.

In allen Erhebungswellen zeigen sich Unterschiede zwischen Altersgruppen bei der engagementbezogenen Internetnutzung (Abbildung 11-3). Diese Unterschiede sind jedoch 2019 deutlich geringer als im Jahr 2004. So betrug im Jahr 2004 beispielsweise der Unterschied zwischen den Altersgruppen der 30- bis 49-Jährigen und den Engagierten ab 65 Jahren 34,4 Prozentpunkte. Dieser Unterschied ist im Jahr 2019 auf 13,7 Prozentpunkte gesunken. Bei den 30- bis 49-Jährigen sowie den 50- bis 64-Jährigen gab es zwischen 2004 und 2009 einen markanten Sprung und danach eine gewisse Stabilität im Anteil der Engagierten, die das Internet nutzen. Im Gegensatz dazu ist bei den Menschen, die 65 Jahre und älter sind, die Internetnutzung seit 2004 deutlich angestiegen. Der Zuwachs von 35,2 Prozentpunkten von 2004 bis 2019 in dieser Gruppe ist maßgeblich dafür verantwortlich, dass sich die Unterschiede in der Nutzung zwischen den Altersgruppen verringert haben. Im Jahr 2019 nutzt fast die Hälfte aller Engagierten ab 65 Jahren das Internet für ihre freiwilligen Tätigkeiten.

Abbildung 11-3 Anteile freiwillig engagierter Personen, die das Internet für ihre freiwillige Tätigkeit nutzen, nach Alter, im Zeitvergleich 2004-2019 (Basis: alle Engagierten)

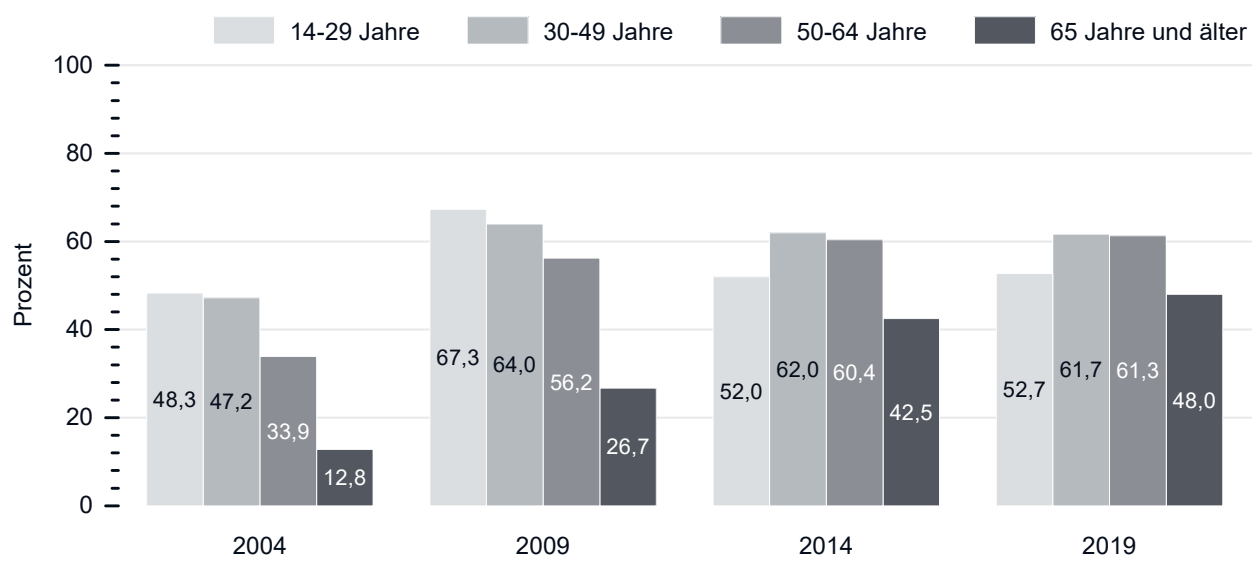

Quelle: FWS, gewichtet, eigene Berechnungen (DZA). Basis: alle Engagierten. FWS 2004 ( $n=5.131)$, FWS 2009 ( $n=6.991)$, FWS 2014 ( $n=12.421$ ), FWS 2019 ( $n=11.925$ ). Im Jahr 1999 wurde Internetnutzung für das freiwillige Engagement nicht erhoben.

Unterschiede zwischen Wellen:

14-29 Jahre: Statistisch signifikante Unterschiede nur zwischen 2009 zu allen andren Jahren.

30-49 Jahre: Statistisch signifikante Unterschiede nur zwischen 2004 zu allen andren Jahren.

50-64 Jahre: Statistisch signifikante Unterschiede nur zwischen 2004 zu allen andren Jahren sowie zwischen 2009 und 2019.

65 Jahre und älter: Unterschiede sind zwischen allen Jahren statistisch signifikant.

Unterschiede zwischen Altersgruppen:

2004 und 2009: Die Gruppen 50-64 Jahre und 65 Jahre und älter unterscheiden sich statistisch signifikant von allen anderen Gruppen. Die Gruppen 14-29 Jahre und 30-49 Jahre unterscheiden sich nicht statistisch signifikant voneinander.

2014 und 2019: Die Gruppen 14-29 Jahre und 65 Jahre und älter unterscheiden sich statistisch signifikant von allen anderen Gruppen. Die

Gruppen 30-49 Jahre und 50-64 Jahre unterscheiden sich nicht statistisch signifikant voneinander. 
Die Bildungsunterschiede bei der Nutzung des Internets für die freiwillige Tätigkeit sind groß und haben sich seit 2004 nicht wesentlich verändert. Im Jahr 2004 nutzten 24,0 Prozent der Menschen mit niedriger Bildung und 53,6 Prozent der Menschen mit hoher Bildung das Internet für ihre freiwillige Tätigkeit (Unterschied: 29,6 Prozentpunkte). Im Jahr 2019 sind es 45,o Prozent der Menschen mit niedriger Bildung und 66,o Prozent der Menschen mit hoher Bildung (Unterschied: 21,o Prozentpunkte). Bei den betrachteten drei Bildungsgruppen (niedrige, mittlere und hohe Bildung) gibt es zwischen 2004 und 2009 jeweils eine deutliche Zunahme der Internetnutzung, wobei danach eine Konsolidierung festzustellen ist. Im Gegensatz dazu ist der Trend der Internetnutzung im Rahmen der freiwilligen Tätigkeit bei Personen, die noch zur Schule gehen, unregelmäßig: Zwischen den Jahren 2004 und 2009 gab es eine statistisch signifikante Zunahme, zwischen den Jahren 2009 und 2014 einen statistisch signifikanten Abfall und zwischen den Jahren 2014 und 2019 besteht kein statistisch signifikanter Unterschied.

Abbildung 11-4 Anteile freiwillig engagierter Personen, die das Internet für ihre freiwillige Tätigkeit nutzen, nach Bildung, im Zeitvergleich 2004-2019 (Basis: alle Engagierten)

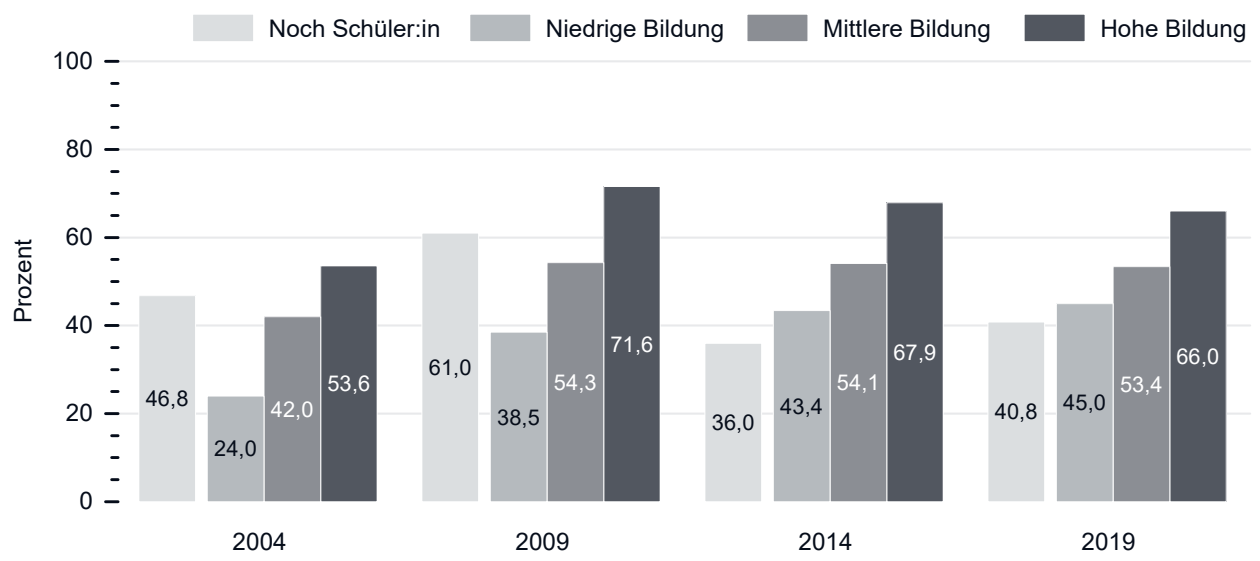

Quelle: FWS, gewichtet, eigene Berechnungen (DZA). Basis: alle Engagierten. FWS 2004 ( $n=5.137)$, FWS 2009 ( $n=6.991)$, FWS 2014 ( $n=12.418$ ), FWS 2019 ( $n$ =11.924). Im Jahr 1999 wurde Internetnutzung für das freiwillige Engagement nicht erhoben.

Unterschiede zwischen Wellen:

Schüler:innen: Die Unterschiede zwischen 2004 und 2019 sowie zwischen 2014 und 2019 sind nicht statistisch signifikant; alle anderen Unterschiede sind statistisch signifikant.

Niedrige Bildung: 2004 unterscheidet sich statistisch signifikant von 2009, 2014 und 2019. 2009 unterscheidet sich statistisch signifikant von 2019. Alle anderen Unterschiede sind nicht statistisch signifikant.

Mittlere Bildung: 2004 unterscheidet sich statistisch signifikant von 2009, 2014 und 2019. Alle anderen Unterschiede sind nicht statistisch signifikant.

Hohe Bildung: 2004 und 2009 unterscheiden sich statistisch signifikant von allen anderen Wellen. Der Unterschied zwischen 2014 und 2019 ist nicht statistisch signifikant.

Unterschiede zwischen Bildungsgruppen:

Niedrige, mittlere und hohe Bildung unterscheiden sich 2004, 2009, 2014 und 2019 statistisch signifikant voneinander.

Schüler:innen unterscheiden sich 2004 statistisch signifikant von niedriger Bildung, 2009 und 2014 von allen drei Bildungsgruppen sowie

2019 von mittlerer und hoher Bildung. 


\subsection{Intensität der Nutzung des Internets für die freiwillige Tätigkeit}

Die Mehrzahl aller freiwillig engagierten Personen gibt an, dass ihre freiwillige Tätigkeit nur teilweise im Internet stattfindet (im Jahr 2014: 52,7 Prozent, im Jahr 2019: 53,6 Prozent; siehe Abbildung 11-5). Für die meisten freiwillig Engagierten, die für ihre Tätigkeit das Internet nutzen, ist dies also eine Unterstützung ihrer freiwilligen Tätigkeit. Nur eine kleine Minderheit gibt in den Jahren 2014 und 2019 an, dass ihre Tätigkeit überwiegend oder ausschließlich im Internet stattfindet: Sowohl 2014 als auch 2019 sind es 2,6 Prozent aller Engagierten. Reines ,Internet-Engagement` wird also nur von einem kleinen Teil der freiwillig Engagierten ausgeübt.

Abbildung 11-5 Intensität der Internetnutzung für die freiwillige Tätigkeit im Zeitvergleich 2014-2019 (Basis: alle Engagierten)

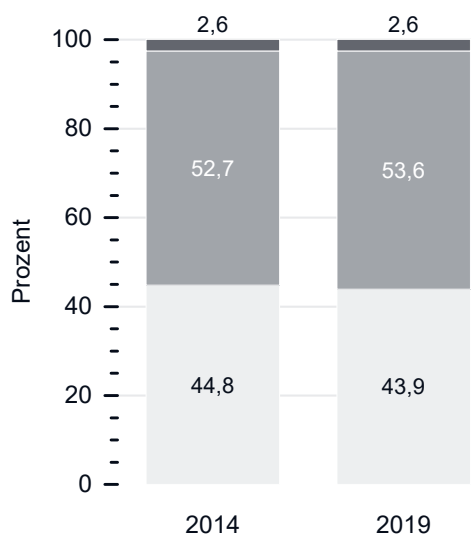

Tätigkeit findet ausschließlich oder überwiegend im Internet statt

Tätigkeit findet teilweise im Internet statt Internet wird für freiwillige Tätigkeit nicht genutzt

Quelle: FWS, gewichtet, eigene Berechnungen (DZA). Basis: alle Engagierten. FWS 2014 ( $n=12.253)$, FWS $2019(n=11.651)$.

Hinweis: Abweichungen in den Prozentwerten ergeben sich durch das Auf- und Abrunden der Werte. Differenzen zu anderen Abbildungen ergeben sich aufgrund fehlender Werte.

Die Unterschiede zwischen 2014 und 2019 sind nicht statistisch signifikant.

Vergleicht man Frauen und Männer hinsichtlich der Rolle, die das Internet für ihre freiwillige Tätigkeit spielt, so finden sich ähnliche Ergebnismuster mit statistisch signifikanten Unterschieden in allen drei Kategorien (Abbildung 11-6). So ist der Anteil der freiwillig Engagierten, die das Internet nicht nutzen, bei Frauen deutlich größer (47,0 Prozent) als bei Männern (40,7 Prozent). Die ,Internet-Engagierten', deren Tätigkeit überwiegend oder ausschließlich im Internet stattfindet, sind aber sowohl bei engagierten Frauen als auch bei engagierten Männern die Minderheit (Frauen: 2,1 Prozent, Männer: 3,o Prozent). 
Abbildung 11-6 Intensität der Internetnutzung für die freiwillige Tätigkeit 2019, gesamt, nach Geschlecht und nach Alter (Basis: alle Engagierten)

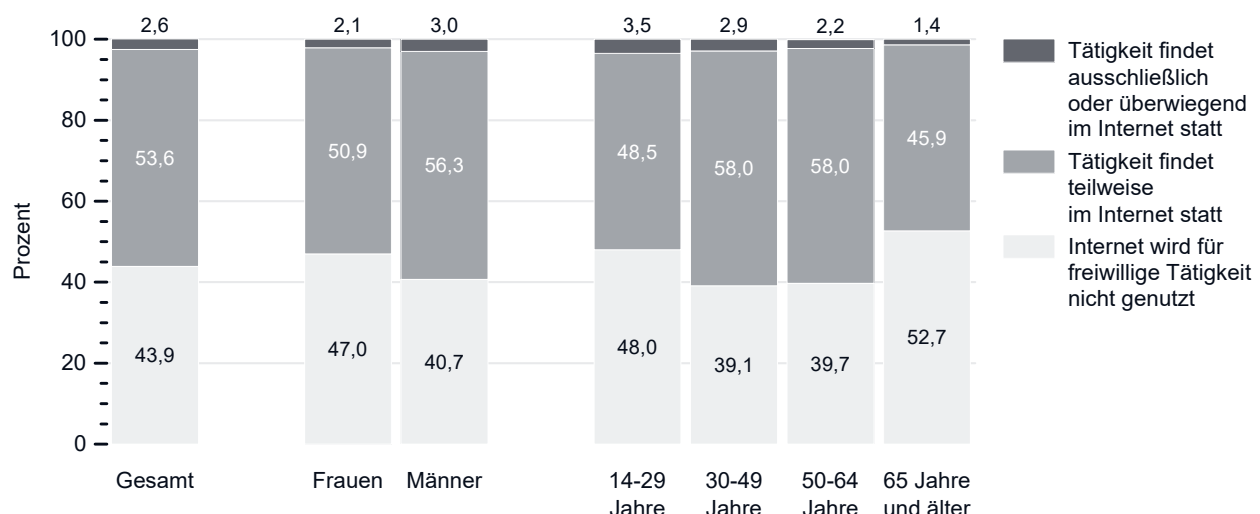

Quelle: FWS, gewichtet, eigene Berechnungen (DZA). Basis: alle Engagierten. FWS 2019 ( $n=11.651)$.

Hinweis: Abweichungen in den Prozentwerten ergeben sich durch das Auf- und Abrunden der Werte. Differenzen zu anderen Abbildungen ergeben sich aufgrund fehlender Werte.

Frauen und Männer unterscheiden sich signifikant in allen Kategorien.

Altersgruppen: In der Kategorie, Internet wird nicht genutzt' ist nur der Unterschied zwischen 30-49 Jahre und 50-64 Jahre statistisch nicht signifikant.

In der Kategorie, teilweise' ist der Unterschied zwischen 14-29 Jahre und 65 Jahre und älter sowie der Unterschied zwischen 30-49 Jahre und 50-64 Jahre nicht statistisch signifikant; alle anderen Unterschiede sind statistisch signifikant.

In der Kategorie,überwiegend' sind nur die Unterschiede von 65 Jahre und älter zu allen andern Altersgruppen statistisch signifikant.

Mit Blick auf das Lebensalter gibt es ebenfalls statistisch signifikante Unterschiede, die sich zwischen den Altersgruppen zeigen (Abbildung 11-6). Bei der sehr intensiven Nutzung, bei der die freiwillige Tätigkeit entweder ausschließlich oder überwiegend im Internet ausgeübt wird, zeigt sich ein klarer Alterseffekt, allerdings auf niedrigem Niveau: Der größte Anteil von Personen, deren freiwillige Tätigkeit vorwiegend oder ausschließlich im Internet stattfindet, findet sich mit 3,5 Prozent in der jüngsten Altersgruppe (14 bis 29 Jahre), der niedrigste Anteil mit 1,4 Prozent bei den Menschen, die 65 Jahre und älter sind. Angesichts der häufig geäußerten Ansicht, dass ältere Menschen das Internet nur mit geringer Intensität nutzen, ist von Interesse, dass es auch in der ältesten Altersgruppe ,Internet-Engagierte' gibt, deren Tätigkeit überwiegend oder ausschließlich im Internet stattfindet (hierzu auch Kersting 2020).

Die Intensität der Internetnutzung für die freiwillige Tätigkeit hängt zudem deutlich mit dem Bildungsstand zusammen. Je höher die Bildung ist, desto anteilig häufiger wird das Internet für die freiwillige Tätigkeit genutzt - und desto intensiver ist die Nutzung (Abbildung 11-7). Von den freiwillig Engagierten mit hoher Bildung geben 3,1 Prozent an, dass ihre freiwillige Tätigkeit ausschließlich oder überwiegend im Internet stattfindet. Bei den freiwillig Engagierten mit niedriger Bildung sind es 1,9 Prozent. Die Anteile der Personen, die noch zur Schule gehen, sind mit denen der Personen mit niedriger Bildung vergleichbar. 
Abbildung 11-7 Intensität der Internetnutzung für die freiwillige Tätigkeit 2019, nach Bildung (Basis: alle Engagierten)

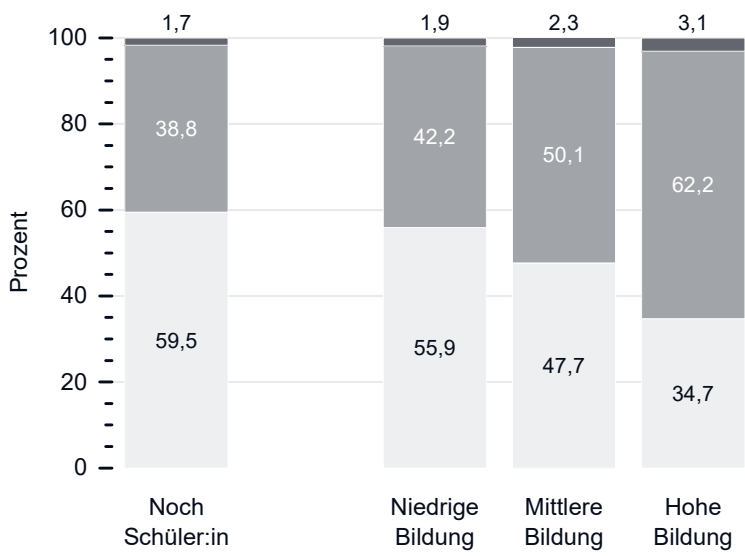

Tätigkeit findet ausschließlich oder überwiegend im Internet statt Tätigkeit findet teilweise im Internet statt Internet wird für freiwillige Tätigkeit nicht genutzt

Quelle: FWS, gewichtet, eigene Berechnungen (DZA). Basis: alle Engagierten. FWS 2019 ( $n=11.650)$.

Hinweis: Abweichungen in den Prozentwerten ergeben sich durch das Auf- und Abrunden der Werte. Differenzen zu anderen Abbildungen ergeben sich aufgrund fehlender Werte.

Für die Kategorien , Internet wird nicht genutzt' und, teilweise' ist jeweils nur der Unterschied zwischen Schüler:innen und niedriger Bildung statistisch nicht signifikant; alle anderen Unterschiede sind statistisch signifikant.

In den Kategorien, ausschließlich oder überwiegend' gibt es keine statistisch signifikanten Unterschiede.

\subsection{Formen der Nutzung des Internets für die freiwillige Tätigkeit}

Das Internet bietet für freiwillig Engagierte die unterschiedlichsten Möglichkeiten der Verwendung. Im Freiwilligensurvey 2019 werden Engagierte, die das Internet für ihre freiwillige Tätigkeit nutzen, nach fünf Formen der aktiv-gestaltenden Internetnutzung gefragt: Beteiligung an sozialen Netzwerken, Blogs, Foren oder Wikis; Erstellen von Newslettern oder Onlineberichten; Betreuung der Homepage eines Vereins oder einer Organisation; Werbung von Geld- oder Sachspenden oder Engagierten (Fundoder Friendraising); sowie Angebot der Lehre, Beratung oder Expertise.

Etwa die Hälfte der Engagierten, die das Internet für ihre freiwillige Tätigkeit nutzen (53,9 Prozent), gibt an, eine oder mehrere der fünf genannten aktiv-gestaltenden Formen der Internetnutzung in ihrer freiwilligen Tätigkeit zu verwenden (Abbildung 11-8). Diese Gruppe teilt sich folgendermaßen auf: 59,1 Prozent der Engagierten, die mindestens eine aktiv-gestaltende Internetnutzungsform verwenden, nutzen genau eine dieser Formen; 26,7 Prozent zwei Formen; 10,0 Prozent drei Formen; 3,5 Prozent vier Formen und o,6 Prozent fünf Formen (ohne Abbildung). Dementsprechend nutzen 46,1 Prozent der Engagierten, die das Internet in ihrer freiwilligen Tätigkeit nutzen, keine der fünf genannten aktiv-gestaltenden Nutzungsformen. Möglicherweise nutzen diese Personen für ihre freiwillige Tätigkeit einfache Internet-Anwendungen (zum Beispiel Versand von E-Mails, Recherche nach Informationen). 
Abbildung 11-8 Anteile freiwillig engagierter Personen, die mindestens eine von fünf aktivgestaltenden Formen der Internetnutzung für die freiwillige Tätigkeit nennen, 2019, a) gesamt, nach Geschlecht und nach Alter, b) nach Bildung (Basis: alle Engagierten, die das Internet nutzen)

a) gesamt, nach Geschlecht und nach Alter

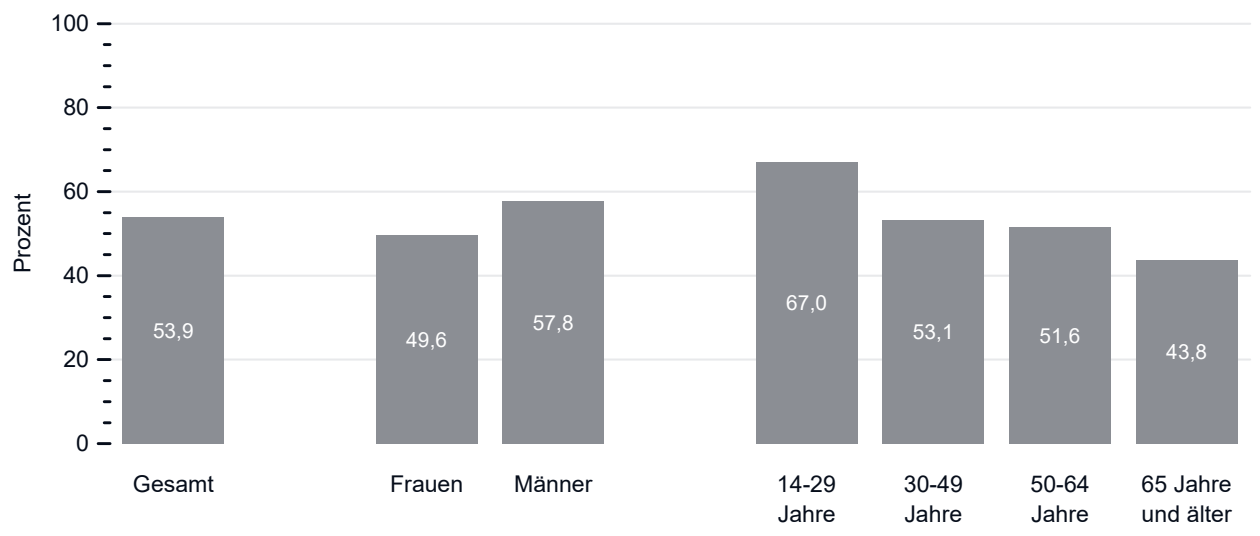

b) nach Bildung

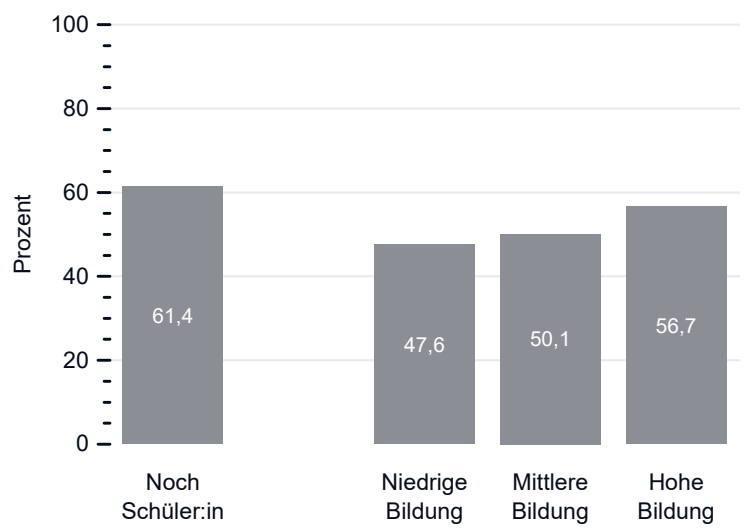

Quelle: FWS, gewichtet, eigene Berechnungen (DZA). Basis: alle Engagierten, die das Internet nutzen. FWS 2019 ( $n=7.213$ ).

Der Geschlechterunterschied ist statistisch signifikant.

Bei den Altersgruppen ist nur der Unterschied zwischen 30-49 Jahre und 50-64 Jahre statistisch nicht signifikant; alle anderen Unterschiede sind statistisch signifikant.

Für die Bildung sind nur die Unterschiede zwischen Schüler:innen und hoher Bildung sowie zwischen niedriger und mittlerer Bildung nicht statistisch signifikant; alle anderen Unterschiede sind statistisch signifikant. 
Männer nutzen die fünf aufgeführten Formen der aktiv-gestaltende Internetnutzung anteilig häufiger als Frauen (Männer: 57,8 Prozent, Frauen: 49,6 Prozent; siehe Abbildung 11-8a). Anteilig am häufigsten werden aktiv-gestaltende Online-Aktivitäten von der jüngsten Altersgruppe (14 bis 29 Jahre) genutzt. Die Gruppen der 30- bis 49-Jährigen sowie der 50- bis 64-Jährigen unterscheiden sich hierbei nicht statistisch signifikant. Bei der ältesten Altersgruppe der 65-Jährigen und Älteren ist der Anteil der Engagierten mit aktiv-gestaltender Internetnutzung am geringsten (Abbildung 11-8a). Ebenfalls deutliche Unterschiede finden sich mit Blick auf den Bildungsstand (Abbildung 11-8b): Engagierte mit niedriger und mittlerer Bildung, die das Internet für ihre freiwillige Tätigkeit nutzen, verwenden anteilig seltener die aktiv-gestaltenden Formen der Internetnutzung für ihre freiwillige Tätigkeit als Engagierte mit hoher Bildung sowie Personen, die noch die Schule besuchen.

Unter den aktiv-gestaltenden Formen der Internetnutzung wird die Beteiligung an sozialen Netzwerken und Blogs am häufigsten genannt. 34,3 Prozent aller Engagierten, die das Internet für ihre freiwillige Tätigkeit nutzen, geben an, das Internet in dieser Form für ihr Engagement zu nutzen (Abbildung 11-9). Die zweithäufigste Nutzungsform ist das Erstellen von Newslettern und Onlineberichten (20,3 Prozent). 13,5 Prozent betreuen die Homepage ihrer Organisation und 12,5 Prozent nutzen das Internet, um Spenden oder Engagierte zu werben. Anteilig am seltensten bieten freiwillig Engagierte, die das Internet für ihre Tätigkeit nutzen, Lehre oder Beratung im Internet an (5,4 Prozent). Zu beachten ist bei der Interpretation dieser Ergebnisse, dass - wie oben bereits dargestellt - Mehrfachnennungen möglich waren (die En-

Abbildung 11-9 Anteile freiwillig engagierter Personen, die das Internet in der angegebenen Form für ihre freiwillige Tätigkeit nutzen, 2019 (Basis: alle Engagierten, die das Internet nutzen; Mehrfachnennungen möglich)

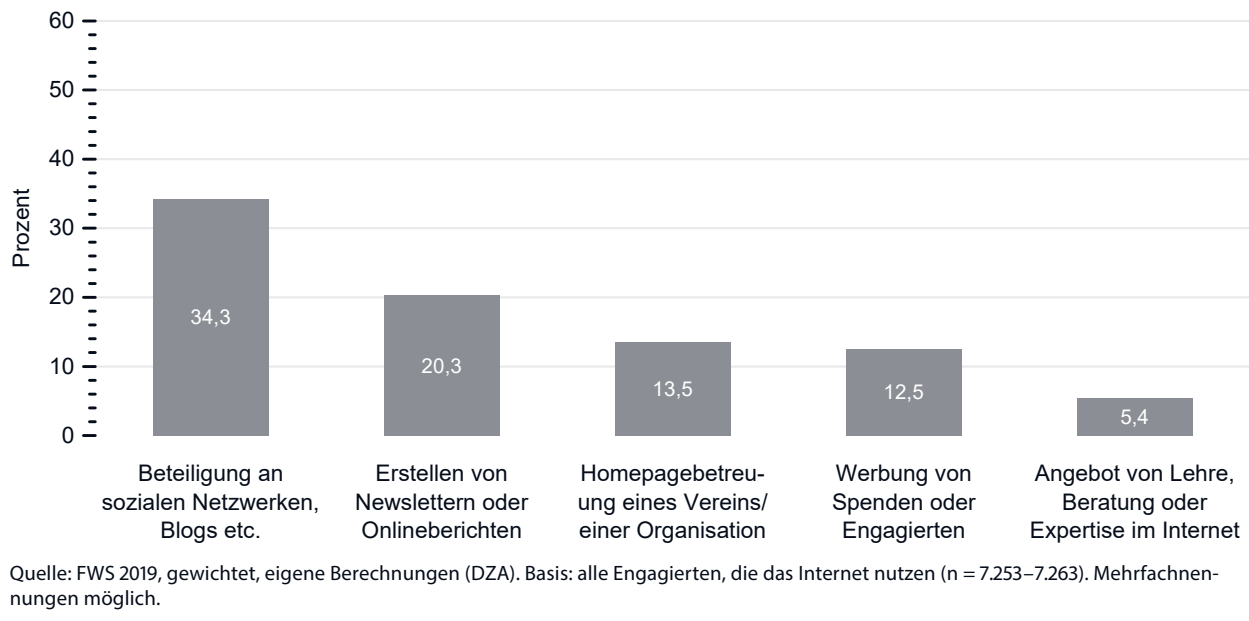


gagierten konnten also mehrere Formen der Internetnutzung angeben) und dass es auch möglich war, keine der angegebenen Nutzungsformen zu nennen.

Fast alle Nutzungsformen werden von männlichen Engagierten, die das Internet für ihre freiwillige Tätigkeit nutzen, anteilig häufiger angegeben als von weiblichen (Abbildung 11-10). Die Geschlechterunterschiede sind insbesondere in den folgenden Bereichen sehr deutlich ausgeprägt: Newsletter-Erstellung (Frauen: 16,5 Prozent, Männer: 23,7 Prozent), Homepagebetreuung (Frauen: 9,6 Prozent, Männer: 17,1 Prozent), im Internet angebotene Lehr- und Beratungsangebote (Frauen: 4,2 Prozent, Männer: 6,4 Prozent). Auch im Bereich soziale Netzwerke gibt es einen statistisch signifikanten Unterschied zwischen den Geschlechtern, der aber relativ gering ausgeprägt ist. Bei der Nutzung des Internets für Werbung von Spenden oder Engagierten gibt es keinen statistisch signifikanten Geschlechterunterschied.

Altersunterschiede in der Verwendung aktiv-gestaltender Formen der Internetnutzung finden sich insbesondere im Bereich der sozialen Netzwerke. Die Anteile der Engagierten, die sich im Kontext ihrer freiwilligen Tätigkeit an sozialen Netzwerken beteiligen, zeigen einen deutlichen Alterseffekt (Abbildung 11-11). Freiwillig Engagierte im Alter zwischen 14 und 29 Jahren geben diese Form der Internutzung anteilig am häufigsten an (53,4 Prozent), die älteste Altersgruppe (65 Jahre und älter) zeigt in dieser Form der Internetnutzung im Vergleich mit den anderen Altersgruppen die geringste Beteiligung (17,o Prozent). Bei der Erstellung von Newslettern und der Betreuung von Websites gibt es fast keine statistisch signifikanten Unterschiede zwischen den Altersgruppen. Die älteste Gruppe von Engagierten nutzt das Internet jedoch weniger als alle anderen Altersgruppen zur Werbung von Spenden und Mitgliedern. Die jüngeren Gruppen unterscheiden sich in dieser Hinsicht nicht statistisch signifikant voneinander. Das Internet für Lehre und Beratungsangebote zu nutzen, wird am häufigsten von der Altersgruppe der 14- bis 29-Jährigen angegeben, allerdings ist auf Grund der kleinen Fallzahlen nur der Unterschied zu der Gruppe der 50- bis 64-Jährigen statistisch signifikant.

Die Unterschiede zwischen den Bildungsgruppen zeigen in diesem Kontext nicht in allen Formen der Internetnutzung einen ansteigenden Bildungseffekt (Abbildung 11-12). In den Kategorien Beteiligung an sozialen Netzwerken, Erstellen von Newslettern und Werbung von Spenden zeigt sich ein ansteigender Bildungseffekt: Die Anteile von Personen mit hoher Bildung sind jeweils höher als die von Personen mit niedriger Bildung. Die Bildungsunterschiede in den Kategorien Homepage-Betreuung und Angebot von Lehre zeigen dieses Muster nicht. So liegen beispielsweise im Bereich der Homepagebetreuung Menschen mit niedriger Bildung (14,6 Prozent) etwa gleichauf mit Personen mit hoher Bildung (14,7 Prozent). 
Abbildung 11-10 Anteile freiwillig engagierter Personen, welche verschiedene Formen der Internetnutzung für die freiwillige Tätigkeit nennen, 2019, nach Geschlecht (Basis: alle Engagierten, die das Internet nutzen; Mehrfachnennungen möglich)

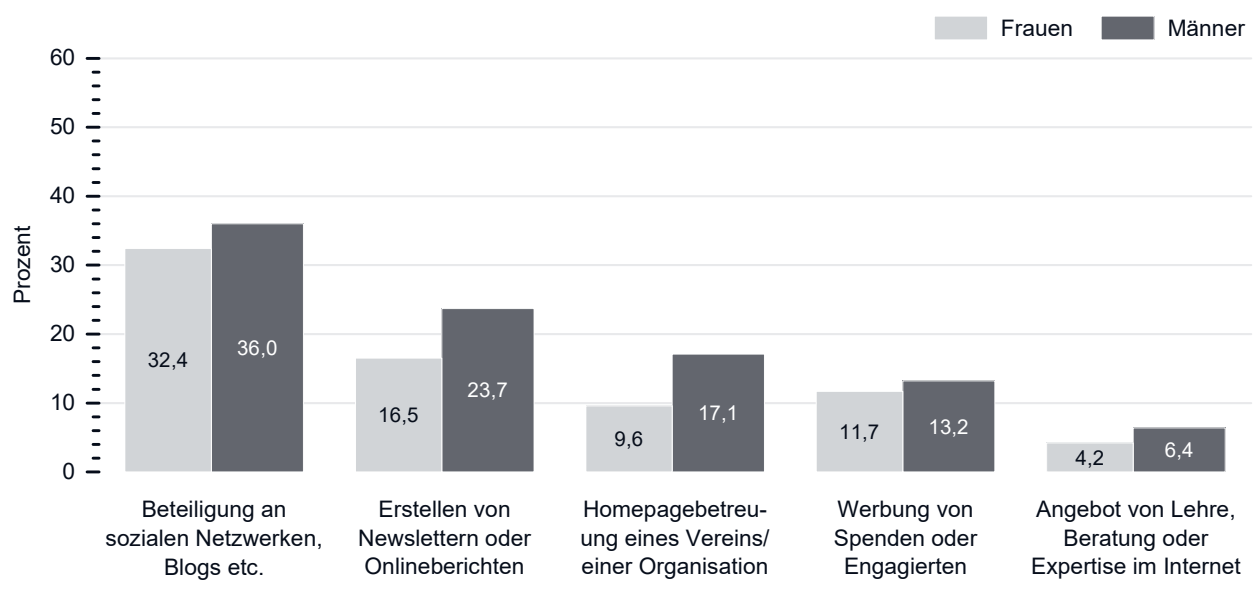

Quelle: FWS, gewichtet, eigene Berechnungen (DZA). Basis: alle Engagierten, die das Internet nutzen. FWS 2019 ( $n=7.253-7.263$ ).

Alle Geschlechterunterschiede sind statistisch signifikant, abgesehen vom Unterschied in der Nutzungsform Werbung von Spenden, der nicht statistisch signifikant ist.

Abbildung 11-11 Anteile freiwillig engagierter Personen, welche verschiedene Formen der Internetnutzung für die freiwillige Tätigkeit nennen, 2019, nach Alter (Basis: alle Engagierten, die das Internet nutzen; Mehrfachnennungen möglich)

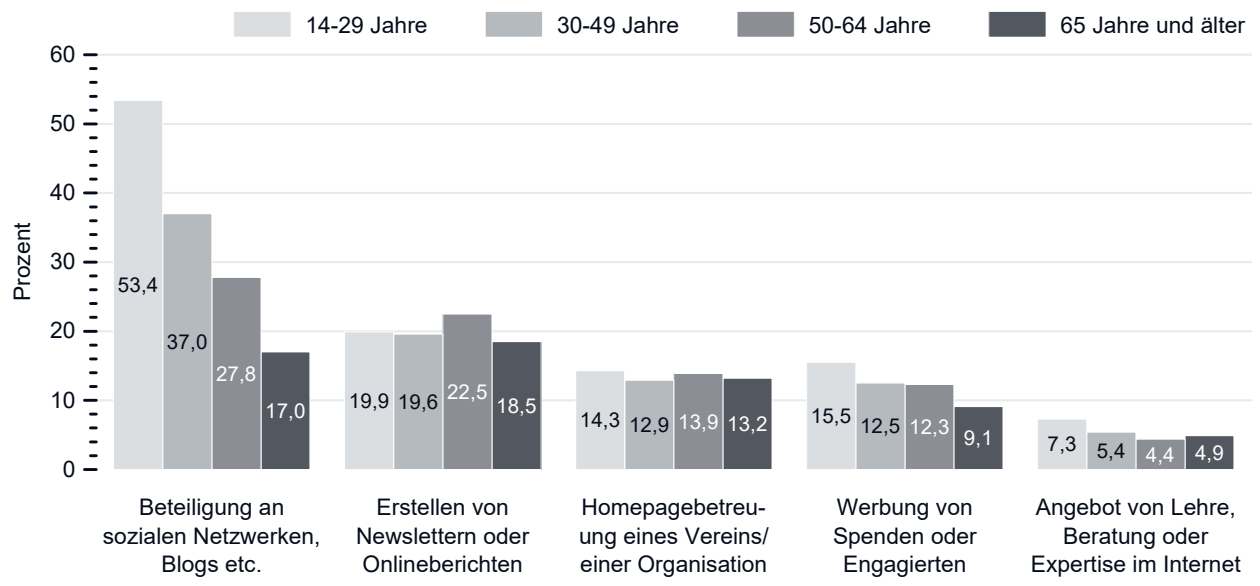

Quelle: FWS, gewichtet, eigene Berechnungen (DZA). Basis: alle Engagierten, die das Internet nutzen. FWS 2019 ( $n=7.253-7.263$ ).

Soziale Netzwerke: Alle Altersgruppen unterscheiden sich statistisch signifikant voneinander.

Newsletter: Nur der Unterschied zwischen 50-64 Jahre und 65 Jahre und älter ist statistisch signifikant.

Homepagebetreuung: Keine statisch signifikanten Unterschiede.

Werbung von Spenden: 65 Jahre und älter unterscheidet sich statistisch signifikant von allen anderen Altersgruppen.

Angebot von Lehre: Nur der Unterschied zwischen 14-29 Jahre und 50-64 Jahre ist statistisch signifikant. 
Abbildung 11-12 Anteile freiwillig engagierter Personen, welche verschiedene Formen der Internetnutzung für die freiwillige Tätigkeit nennen, 2019, nach Bildung (Basis: alle Engagierten, die das Internet nutzen; Mehrfachnennungen möglich)

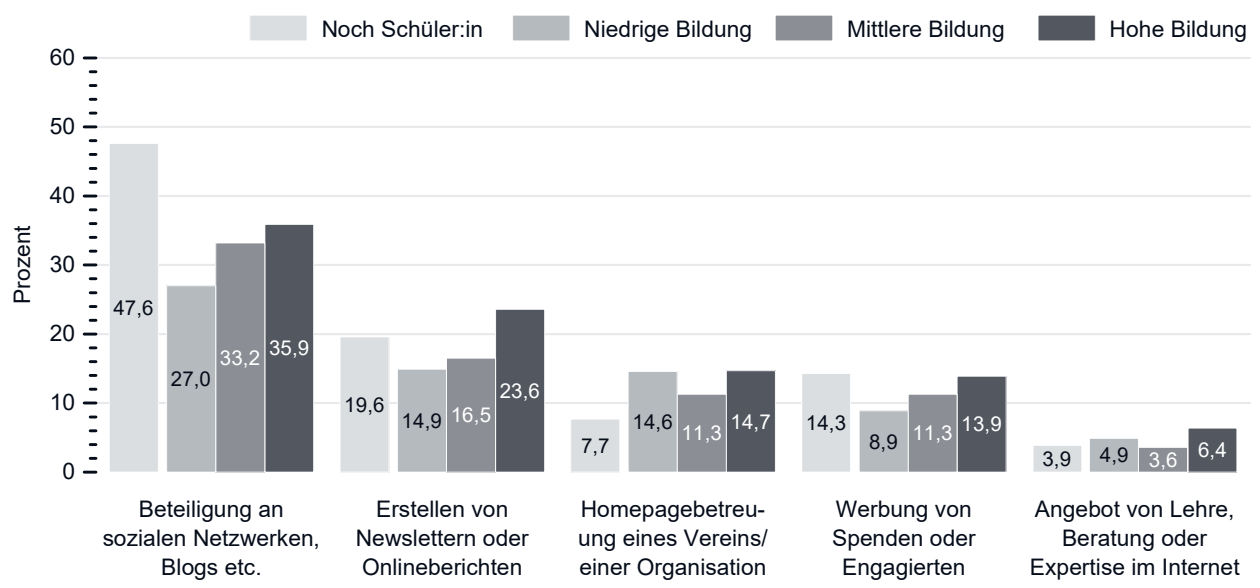

Quelle: FWS, gewichtet, eigene Berechnungen (DZA). Basis: alle Engagierten, die das Internet nutzen. FWS 2019 ( $n=7.253-7.263$ ).

Soziale Netzwerke: Alle Unterschiede sind statistisch signifikant, mit Ausnahme des Unterschieds zwischen mittlerer Bildung und hoher Bildung, der nicht statistisch signifikant ist.

Newsletter: Nur die Unterschiede zwischen hoher Bildung und sowohl mittlerer als auch niedriger Bildung sind statistisch signifikant. Homepagebetreuung: Nur die Unterschiede zwischen hoher Bildung und mittlerer Bildung sowie hoher Bildung und Schüler:innen sind statistisch signifikant.

Werbung von Spenden: Nur der Unterschied zwischen hoher Bildung und niedriger Bildung ist statistisch signifikant.

Angebot von Lehre: Nur der Unterschied zwischen hoher Bildung und mittlerer Bildung ist statistisch signifikant.

Besonders interessant ist die Antwort auf die Frage, ob Personen, die sich in der Intensität der Internetnutzung unterscheiden, auch verschiedene Formen der Internetnutzung betreiben. Auch hier erfolgt ein Vergleich nur innerhalb der Gruppe der Personen, die das Internet für ihre freiwillige Tätigkeit nutzen. In Abbildung 11-13 sind die Unterschiede in den Formen der Internetnutzung für zwei Gruppen von Engagierten dargestellt. Die erste Gruppe umfasst die Personen, deren Tätigkeit teilweise im Internet stattfindet; die zweite Gruppe umfasst die Personen, deren Tätigkeit ausschließlich oder überwiegend im Internet stattfindet. Engagierte, die überwiegend oder ausschließlich das Internet für ihre freiwillige Tätigkeit nutzen, verwenden alle Formen der Internetnutzung anteilig häufiger als Engagierte, die nur teilweise das Internet für ihre Tätigkeit nutzen (Abbildung 11-13). Dies gilt für alle Kategorien: die Beteiligung an sozialen Netzwerken (33,7 Prozent zu 59,1 Prozent), das Erstellen von Newslettern (20,3 Prozent zu 29,5 Prozent), die Betreuung von Websites (13,1 Prozent zu 30,5 Prozent), die Werbung von Spenden (12,4 Prozent zu 19,o Prozent) sowie das Angebot von Lehre (5,o Prozent zu 14,2 Prozent). 
Abbildung 11-13 Anteile freiwillig engagierter Personen, welche verschiedene Formen der Internetnutzung für die freiwillige Tätigkeit nennen, 2019, nach Nutzungsintensität (Basis: alle Engagierten, die das Internet nutzen; Mehrfachnennungen möglich)

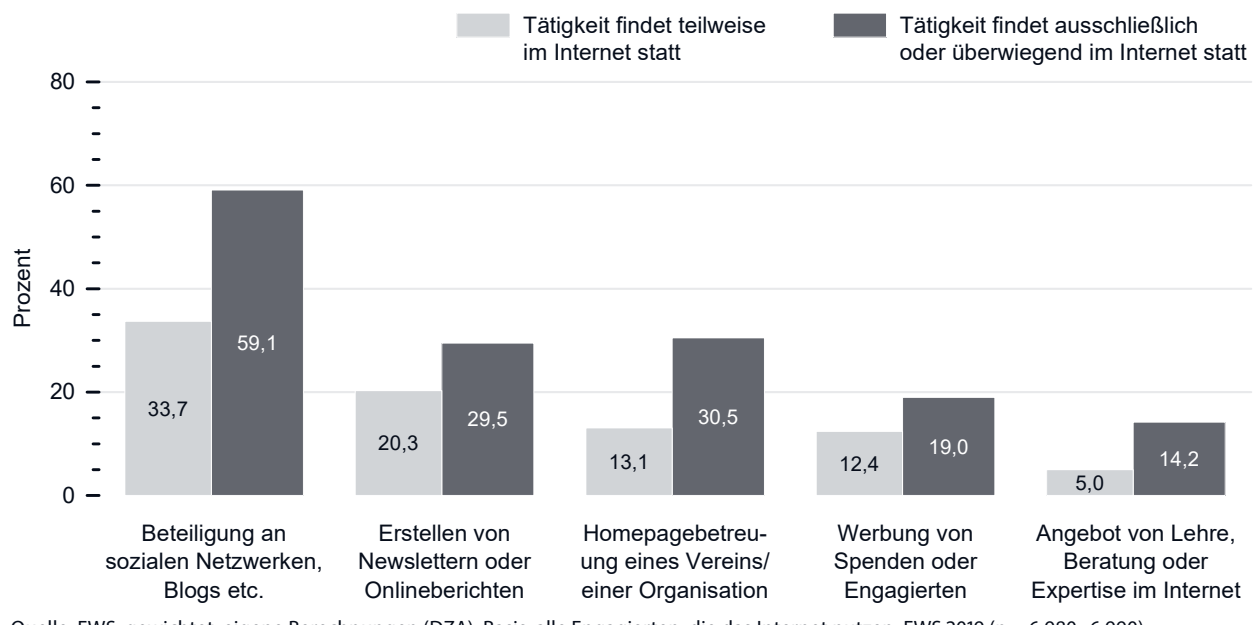

Quelle: FWS, gewichtet, eigene Berechnungen (DZA). Basis: alle Engagierten, die das Internet nutzen. FWS 2019 ( $n=6.980-6.990)$.

Die Unterschiede zwischen den Gruppen,Tätigkeit findet teilweise im Internet statt' und,Tätigkeit findet ausschließlich oder überwiegend im Internet statt' sind für alle fünf Nutzungsformen statistisch signifikant.

\subsection{Fazit}

Das Internet und die damit verknüpften Möglichkeiten der Information, Kommunikation und Organisation spielen eine große Rolle im freiwilligen Engagement. Die Mehrheit der Engagierten nutzt das Internet im Rahmen der freiwilligen Tätigkeit. Überraschend sind aber zwei Befunde: Erstens ist der Anteil der Engagierten, die im Rahmen ihrer freiwilligen Tätigkeit das Internet nutzen, nur von $2004 \mathrm{Zu} 2009$ angestiegen, seitdem aber recht stabil geblieben. Zweitens nutzen nicht alle Engagierten das Internet für ihre freiwillige Tätigkeit, sondern nur etwas mehr als die Hälfte, je nach Jahr zwischen 55 Prozent und 57 Prozent. Dies bedeutet auch, dass ein großer Teil der Engagierten (mehr als 40 Prozent) das Internet für ihre Tätigkeit nicht nutzt. Ähnliche Befunde werden, bezogen auf jüngere freiwillig Engagierte, auch im Dritten Engagementbericht dargestellt. Dort heißt es mit Blick auf Jugendliche und junge Erwachsene, dass sich „43,2 Prozent der Befragten als digital Engagierte beschreiben lassen, die ihr Engagement teilweise, überwiegend oder sogar vollständig mittels digitaler Medien ausüben“ (Deutscher Bundestag 2020: 33). Offensichtlich gibt es im freiwilligen Engagement viele Bereiche, in denen Organisation und Kommunikation in , analoger Form' stattfinden.

Allerdings ist anzumerken, dass die Nutzung des Internets für die freiwillige Tätigkeit in der Gruppe der Engagierten, die 65 Jahre und älter sind, von 2004 bis 2019 
um 35,2 Prozentpunkte deutlich zugenommen hat. Diese Gruppe stellt also die Ausnahme von der Regel dar, dass die Nutzung des Internets sich nach 2009 wenig verändert hat. Im Jahr 2019 nutzt fast die Hälfte aller Engagierten im Ruhestandsalter das Internet für ihre freiwillige Tätigkeit.

Zudem ist es bei der Interpretation der Ergebnisse wichtig zu berücksichtigen, dass sich über die Zeit die Bedeutung des Begriffs, Internetnutzung' gewandelt hat - und zwar gerade bei Personen aus jüngeren Geburtsjahrgängen, die mit digitalen Technologien aufgewachsen sind. Obwohl bestimmte Tätigkeiten im technischen Sinne die Nutzung des Internets notwendig machen, werden sie möglicherweise nicht als ,Internetnutzung' wahrgenommen. In diesen Fällen werden sie von den Befragten auch nicht berücksichtigt, wenn sie nach der Nutzung des Internets bei der freiwilligen Tätigkeit gefragt werden. Dies könnte insbesondere für die Nutzung von Smartphones gelten, deren Funktionsweise zwar auf dem Internet basiert, die aber im Alltagsverständnis nicht mit dem Internet verknüpft sind. Ein Beispiel dafür ist die weitverbreitete Nutzung von Messenger-Diensten (wie WhatsApp oder Telegram). Aber auch Tätigkeiten wie Microblogging (etwa Twitter), Civic Technology (durch freiwillig Engagierte betriebene Kommunikationsplattformen), Crowdsourcing (Auslagerung von Aufgaben an freiwillig Engagierte) oder Participatory Mapping (Kartographie mit partizipativen Methoden, um das räumliche Wissen lokaler Gemeinschaften aufzuzeichnen) sind hier zu nennen. Hier stehen möglicherweise die Inhalte des freiwilligen Engagements im Mittelpunkt, während die Technik als gegeben vorausgesetzt wird und als nicht nennenswert erscheint. Diesem ,jungen Engagement im digitalen Zeitalter" und den Implikationen für das freiwillige Engagement widmet sich der Dritte Engagementbericht, in dem auf den selbstverständlichen Umgang junger Menschen mit digitalen Medien hingewiesen wird (Deutscher Bundestag 2020: 33).

Die digitale Spaltung der Gesellschaft macht auch vor dem freiwilligen Engagement nicht halt. Bildung und Einkommen einer Person bestimmen nach wie vor die Möglichkeiten des Zugangs und der Nutzung des Internets. So heißt es zwar, dass die Digitalisierung der Gesellschaft insgesamt angestiegen ist, dass aber „niedrig Gebildete in vielen Kompetenzbereichen abgehängt" sind (Initiative D21 e. V. 2020: 10). Wie in anderen Bereichen des gesellschaftlichen Lebens gibt es auch im Bereich des freiwilligen Engagements erhebliche Unterschiede nach Geschlecht, Alter und Bildung in der Nutzung des Internets. Freiwillig engagierte Männer nutzen das Internet anteilig häufiger als freiwillig engagierte Frauen, ältere Engagierte ab 65 Jahren nutzen es anteilig seltener als jüngere Altersgruppen und Engagierte mit hoher Bildung nutzen es anteilig häufiger als Engagierte mit niedriger Bildung. Hier ist es notwendig, dass die Engagementpolitik sowie zivilgesellschaftliche Organisationen nachsteuern. Es sollte für alle freiwillig engagierten Menschen möglich sein, das Internet für ihr Engagement zu nutzen, wenn dies für die ausgeübten Tätigkeiten hilfreich ist, unabhängig von Geschlecht, Alter oder Bildung. Online-Aktivitäten im Rahmen des Engagements eröffnen flexible Möglichkeiten zur Partizipation, insbesondere für die Menschen, die zeitlich, gesundheitlich oder finanziell eingeschränkt sind. 
Das Internet kann für freiwilliges Engagement unterschiedlich bedeutsam sein. ,Online-Volunteering' umfasst freiwilliges Engagement, das die Möglichkeiten des Internets nutzt und unabhängig von Ort und Zeit durchgeführt werden kann (Lewis et al. 2014). Beispiele für dieses, Online-Volunteering' sind die Produktion digitaler Güter wie Enzyklopädien, die internetvermittelte Kommunikation mit anderen freiwillig Engagierten über Chats oder Blogs, die Sammlung von Spenden oder Unterstützungsleistungen sowie die Organisationen von Aktionen, die ohne eine internetbasierte Form der Vernetzung kaum denkbar sind (Ihm 2017). Im Freiwilligensurvey 2019 wird nach fünf ausgewählten Formen der Internetnutzung gefragt: Beteiligung an sozialen Netzwerken, Erstellen von Newslettern, Homepage-Betreuung, Werbung von Spenden sowie Angebote der Beratung. Interessant ist herbei, dass internetaffine freiwillig Engagierte nicht eine Vielzahl von Formen aktiv-gestaltender Internetnutzung angeben, sondern in den meisten Fällen nur eine oder zwei dieser Formen. Bei den aktiv-gestalteten Tätigkeiten wurde die Beteiligung an sozialen Netzwerken anteilig am häufigsten genannt, anteilig am seltensten wurden Angebote der Beratung angegeben. Möglicherweise gibt es auch im Bereich der Internetnutzung im freiwilligen Engagement Spezialisierungen je nach Interesse und Kompetenz.

Freiwilliges Engagement, das überwiegend oder ausschließlich im Internet stattfindet, ist ein vergleichsweise neues Phänomen aber möglicherweise kein Massenphänomen. ,Internet-Engagierte' mögen eine Avantgarde des freiwilligen Engagements sein. Laut der Daten des Freiwilligensurveys scheinen sie aber - wie das bei einer Avantgarde nicht selten der Fall ist - eine kleine Gruppe innerhalb der Engagierten zu bilden. Auch im Jahr 2019 üben nur 2,6 Prozent aller freiwillig engagierten Personen ihre Tätigkeit überwiegend oder ausschließlich im Internet aus. Bei allen anderen freiwilligen Engagierten, die das Internet nutzen, findet die freiwillige Tätigkeit nur teilweise im Internet statt.

Etwa 57 Prozent der Engagierten nutzen das Internet für ihre Tätigkeit. Umgekehrt heißt dies: Das Internet spielt für einen großen Teil der freiwillig Engagierten in Deutschland keine große Rolle. Etwas mehr als 4o Prozent der Engagierten nutzen das Internet für ihre freiwillige Tätigkeit nicht. Möglicherweise spielt hier neben der digitalen Spaltung die digitale Souveränität eine Rolle. Digitale Souveränität kann als Fähigkeit verstanden werden, „digitale Medien selbstbestimmt und unter eigener Kontrolle zu nutzen“ (Blossfeld et al. 2018: 12). Dies bedeutet auch, das Internet nicht um jeden Preis zu nutzen, sondern nur dort, wo es den Zielen des freiwilligen Engagements dient. 


\section{Literatur}

Blossfeld, H.-P., Bos, W., Daniel, H.-D., Hannover, B., Köller, O., Lenzen, D., McElvany, N., Roßbach, H.-G., Seidel, T., Tippelt, R., \& Wößmann, L. (2018). Digitale Souveränität und Bildung. Münster: Waxmann.

Deutscher Bundestag (2020). Dritter Engagementbericht. Zukunft Zivilgesellschaft: Junges Engagement im digitalen Zeitalter und Stellungnahme der Bundesregierung (Drucksache 19/19320). Berlin: Deutscher Bundestag.

Heinze, R. G., Beckmann, F., \& Schönauer, A.-L. (2019). Die Digitalisierung des Engagements: Zwischen Hype und disruptivem Wandel. In: R. G. Heinze, S. Kurtenbach \& J. Überbacker (Hrsg.) Digitalisierung und Nachbarschaft. Erosion des Zusammenlebens oder neue Vergemeinschaftung? (S. 61-90). Baden-Baden: Nomos.

Hinz, U., Wegener, N., Weber, M., \& Fromm, J. (2014). Digitales Bürgerschaftliches Engagement. Berlin: FOKUS. Online: https://www.oeffentliche-it.de/documents/10181/14412/Digitales $+\mathrm{B} \% \mathrm{C}_{3} \% \mathrm{BCrgerschaftliches+Engagement} \mathrm{(zuletzt} \mathrm{abgerufen} \mathrm{11.} \mathrm{02.} \mathrm{2021).}$

Hoffmann, C.P., Lutz, C., \& Poëll, R. (2015). DIVSI-Studie: Beteiligung im Internet: Wer beteiligt sich wie? Hamburg: Deutsches Institut für Vertrauen und Sicherheit im Internet (DIVSI).

Huxhold, O., \& Otte, K. (2019). Internetzugang und Internetnutzung in der zweiten Lebenshälfte (DZA Aktuell Bd. 1/2019). Berlin: Deutsches Zentrum für Altersfragen.

Ihm, J. (2017). Classifying and relating different types of online and offline volunteering. Voluntas: International Journal of Voluntary and Nonprofit Organizations, 28(1), 400-419.

Initiative D21 e. V. (Hrsg.) (2020). D21-Digital-Index 2019/2020. Jährliches Lagebild zur Digitalen Gesellschaft. Berlin: Initiative D21.

Kersting, N. (2020). Digitale Ungleichheiten und digitale Spaltung. In T. Klenk, F. Nullmeier \& G. Wewer (Hrsg.) Handbuch Digitalisierung in Staat und Verwaltung (S. 1-11). Wiesbaden: Springer VS.

Lewis, K., Gray, K., \& Meierhenrich, J. (2014). The structure of online activism. Sociological Science, 1, 1-9.

Statistisches Bundesamt (2019). Private Haushalte in der Informationsgesellschaft - Nutzung von Informations- und Kommunikationstechnologien. Wiesbaden: Statistisches Bundesamt. 